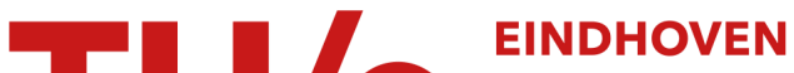 UNIVERSITY OF TECHNOLOGY
}

\section{Magnetic order in the Ising model with parallel dynamics}

\section{Citation for published version (APA):}

Cirillo, E. N. M., Nardi, F. R., \& Polosa, A. D. (2001). Magnetic order in the Ising model with parallel dynamics. Physical Review E - Statistical, Nonlinear, and Soft Matter Physics, 64(5), [057103]. https://doi.org/10.1103/PhysRevE.64.057103

DOI:

10.1103/PhysRevE.64.057103

Document status and date:

Published: 01/01/2001

\section{Document Version:}

Publisher's PDF, also known as Version of Record (includes final page, issue and volume numbers)

\section{Please check the document version of this publication:}

- A submitted manuscript is the version of the article upon submission and before peer-review. There can be important differences between the submitted version and the official published version of record. People interested in the research are advised to contact the author for the final version of the publication, or visit the $\mathrm{DOI}$ to the publisher's website.

- The final author version and the galley proof are versions of the publication after peer review.

- The final published version features the final layout of the paper including the volume, issue and page numbers.

Link to publication

\section{General rights}

Copyright and moral rights for the publications made accessible in the public portal are retained by the authors and/or other copyright owners and it is a condition of accessing publications that users recognise and abide by the legal requirements associated with these rights.

- Users may download and print one copy of any publication from the public portal for the purpose of private study or research.

- You may not further distribute the material or use it for any profit-making activity or commercial gain

- You may freely distribute the URL identifying the publication in the public portal.

If the publication is distributed under the terms of Article $25 \mathrm{fa}$ of the Dutch Copyright Act, indicated by the "Taverne" license above, please follow below link for the End User Agreement:

www.tue.nl/taverne

Take down policy

If you believe that this document breaches copyright please contact us at:

openaccess@tue.nl

providing details and we will investigate your claim. 


\title{
Magnetic order in the Ising model with parallel dynamics
}

\author{
Emilio N. M. Cirillo \\ Dipartimento Me. Mo. Mat., Università degli Studi di Roma 'La Sapienza," via A. Scarpa 16, 00161 Roma, Italy \\ Francesca R. Nardi \\ Eurandom, P.O. Box 513, 5600MB, Eindhoven, The Netherlands \\ Antonio D. Polosa \\ Department of Physics, University of Helsinki, P.O. Box 64, FIN-00014, Helsinki, Finland
}

(Received 27 April 2001; published 24 October 2001)

\begin{abstract}
It is discussed how the equilibrium properties of the Ising model are described by an Hamiltonian with an antiferromagnetic low temperature behavior if only an heat bath dynamics, with the characteristics of a Probabilistic cellular automaton, is assumed to determine the temporal evolution of the system.
\end{abstract}

DOI: 10.1103/PhysRevE.64.057103

PACS number(s): 05.50.+q, 75.10.-b, 64.60.Fr

\section{INTRODUCTION}

In this Brief Report we discuss the equilibrium properties of probabilistic cellular automata (PCA's) reversible with respect to a Gibbs measure derived by a suitable Hamiltonian. A PCA [1-3] is a lattice model with discrete variables that are subject to a probabilistic simultaneous updating in discrete time steps: all configurations are accessible in a single updating. PCA's arise as an extended definition of deterministic cellular automata in which the updating follows a set of deterministic local rules. The huge number of possible deterministic (or probabilistic) rules makes the topic of cellular automata overwhelmingly abundant. One of the most famous cellular automata systems is Conway's "game of life" [4]; in spite of the very simple deterministic majority rule assigned, the system, which is a kind of spin lattice, presents an extremely rich and complex evolution pattern.

PCA's have been studied in a wide variety of contexts, ranging from biology to the theory of automation. These models can be thought of as interacting particle systems: a particular class of cellular automata, known as bootstrap percolation, has been introduced in [5] to model the propagation of cracks in solids (see also [6]). A wide discussion on numerical and rigorous results on bootstrap percolation can be found in [7] and [8]. The connections with statistical mechanics models have been further investigated in [9] and in a number of papers with a mathematical physics cut [10-12]. The particular family of automata we study is obtained by implementing in parallel fashion the heat bath dynamics [11]. In other terms, we define a rule for the transition probabilities such that all single spins of a lattice are updated simultaneously with heat bath rates. This amounts to defining a Markov chain for the evolution of the spin system, having the characteristics of a PCA.

We observe that the way of implementing the heat bath dynamics reflects into a qualitative modification of the equilibrium properties of the model. In particular, an Ising-like ferromagnetic Hamiltonian with two body interactions, defines a PCA reversible with respect to a Gibbs measure determined by an Hamiltonian allowing a low temperature an- tiferromagnetic phase. This behavior is absent if a serial dynamics is implemented, for which at most one spin of the system is updated at any time.

The paper is organized as follows. In Sec. II we define the PCA under consideration and the spin model Hamiltonian, $H$, determining the heat bath single spin rates. In Sec. III the structure and the low temperature antiferromagnetic properties of $H^{\prime}$ are discussed in the case of the standard nearest neighbor Ising model and in the case of the (two body) nextto-nearest neighbor interaction. The dynamical generation of antiferromagnetic couplings is reviewed in the concluding remarks.

\section{COUPLING PROLIFERATION IN REVERSIBLE PCA'S}

Let $\Lambda$ be a finite two-dimensional square lattice and $|\Lambda|$ its cardinality. For each $x=\left(x_{1}, x_{2}\right), y=\left(y_{1}, y_{2}\right) \in \Lambda$ we denote by $|x-y|$ the Euclidean distance on the lattice. Let $\sigma(x) \in\{-1,+1\}$ a spin variable associated to the site $x$ $\in \Lambda$; the space $\{1,-1\}^{\Lambda}$ of configurations is denoted by $\mathcal{S}$. Let us consider a generic Hamiltonian $H(\sigma)$ and the corresponding equilibrium Gibbs measure $\mu(\sigma)$ $=\exp \{-\beta H(\sigma)\} / \Sigma_{\eta \in \mathcal{S}} \exp \{-\beta H(\eta)\}$ with $\beta$ the inverse of the temperature. We now define the heat bath single spin rates: given the site $x \in \Lambda$, we consider the Gibbs equilibrium measure for $\sigma_{x}$ with respect to a fixed configuration $\sigma$ on $\Lambda \backslash\{x\}$. Letting $a \in\{-1,+1\}$, we have

$p_{x}(a \mid \sigma)=\frac{\exp \{-\beta H(a, \sigma)\}}{\exp \{-\beta H(a, \sigma)\}+\exp \{-\beta H(-a, \sigma)\}}$,

where $( \pm a, \sigma)$ are the configurations equal to $\sigma$ on $\Lambda \backslash\{x\}$ and to $\pm a$ on $x$. Note that the normalization condition $p_{x}(a \mid \sigma)+p_{x}(-a \mid \sigma)=1$ is trivially satisfied.

We can now implement the heat bath dynamics in a serial fashion, namely, we can consider the Markov chain $\sigma_{t}(t$ being the discrete time temporal variable), with transition probabilities 


$$
P(\sigma, \eta)=\left\{\begin{array}{l}
(1 /|\Lambda|) p_{x}(\eta(x) \mid \sigma) \text { if } \exists x \in \Lambda \text { such that } \sigma=\eta \text { on } \Lambda \backslash\{x\} \\
0 \quad \text { otherwise }
\end{array}\right.
$$

for all $\sigma, \eta \in \mathcal{S}$. The transition probabilities (2) are reversible with respect to the Gibbs measure, i.e., the detailed balance condition is satisfied or, equivalently, the equilibrium measure is the Gibbs measure.

A different point of view can be taken [11]: we define the transition probabilities $P(\sigma, \eta)$ in such a way that all the spins are simultaneously and independently updated, in a parallel fashion, with the heat bath rates (1). Thus, instead of Eq. (2) we consider the Markov chain $\sigma_{t}$ defined by

$$
P(\sigma, \eta)=\prod_{x \in \Lambda} p_{x}(\eta(x) \mid \sigma) \quad \forall \sigma, \eta \in \mathcal{S} .
$$

This amounts to defining a PCA. In general the equilibrium properties of the Markov chain (3) are not trivial, for instance it is not obvious that there exists a Gibbs measure such that the detailed balance principle is satisfied.

Let us consider, now, the case of the two body interactions and suppose that the Hamiltonian has the form

$$
H(\sigma)=-\sum_{x, y \in \Lambda} J_{x, y} \sigma(x) \sigma(y)-\sum_{x \in \Lambda} h_{x} \sigma(x),
$$

where $J_{x, y} \in \mathrm{R}$ are the pair couplings between spins at sites $x, y$, and $h_{x} \in \mathbb{R}$ is the external magnetic field acting on the spin at site $x$. For physical reasons we suppose that the pair couplings are symmetric, namely $J_{x, y}=J_{y, x}$ for all $x, y \in \Lambda$. The heat bath single spin rates are given by

$$
\begin{aligned}
p_{x}(a \mid \sigma) & =\frac{1}{1+\exp \{-\beta[H(-a, \sigma)-H(a, \sigma)]\}} \\
& =\frac{1}{2}\left[1+a \tanh \beta S_{x}(\sigma)\right],
\end{aligned}
$$

where $S_{x}(\sigma)=\Sigma_{y \in \Lambda \backslash\{x\}} J_{x, y} \sigma(y)+h_{x}$ for any $\sigma \in \mathcal{S}$ and $x$ $\in \Lambda$. It is easy to show [11] that the probabilistic cellular automaton (3) with single spin rates (5) is reversible with respect to the Gibbs measure $\mu^{\prime}$ on $\mathcal{S}$ associated with the Hamiltonian

$$
H^{\prime}(\sigma)=-\beta \sum_{x \in \Lambda} h_{x} \sigma(x)-\sum_{x \in \Lambda} \log \cosh \left[\beta S_{x}(\sigma)\right]
$$

In other words the detailed balance condition $P(\sigma, \eta) \exp \left\{-H^{\prime}(\sigma)\right\}=P(\eta, \sigma) \exp \left\{-H^{\prime}(\eta)\right\}$ is satisfied for any $\sigma, \eta \in \mathcal{S}$. This means that $H^{\prime}(\sigma)$ is the equilibrium Hamiltonian of a system governed by $H(\sigma)$ and evolving with the law (3).

The choice of the two body interaction in Eq. (4) is strictly connected to the reversibility of the resulting probabilistic cellular automaton (3) [2,3]. For example, consider the three body interaction Hamiltonian $H(\sigma)$ $=\sum_{x, y, z \in \Lambda} J_{x, y, x} \sigma(x) \sigma(y) \sigma(z)$ with the three body couplings $J_{x, y, z}$ symmetric with respect to permutations of the indices and such that $J_{x, y, z} \neq 0$ if and only if $x \neq y \neq z \neq x$. Then the problem of showing the reversibility of the parallel dynamics can be reduced to the problem of finding a function $\phi: \sigma$ $\in \mathcal{S} \rightarrow \phi(\sigma) \in \mathbb{R}$ such that

$$
\begin{aligned}
& \phi(\sigma)-3 \beta \eta(x) \sum_{y, z \in \Lambda \backslash\{x\}} J_{x, y, z} \sigma(y) \sigma(z) \\
& =\phi(\eta)-3 \beta \sigma(x) \sum_{y, z \in \Lambda \backslash\{x\}} J_{x, y, z} \eta(y) \eta(z),
\end{aligned}
$$

which has no solution.

Let us now discuss the main feature of reversible heat bath derived probabilistic automata. As it has been seen above, if the starting Hamiltonian is given by Eq. (4), then the Markov chain (3) is reversible with respect to the Gibbs measure with Hamiltonian $H^{\prime}$ given by Eq. (6). It is clear that new kind of interactions, different from the one present in the original Hamiltonian $H$, arise when $H^{\prime}$ is considered.

Suppose, for instance, that the starting Hamiltonian has range $r>0$, namely $J_{x, y}=0$ for any $x, y \in \Lambda$ such that $\mid x$ $-y \mid>r$. Then we have $S_{x}(\sigma)=\Sigma_{y: 0<|x-y| \leqslant r} J_{x, y} \sigma(y)+h_{x}$, a sort of average of the spins inside a ball centered at site $x$ with radius equal to $r$. Hence, by expanding $H^{\prime}$ as a sum of potentials we will get all the possible couplings inside the ball, starting from the two body up to the $N(r)$ body interaction, with $N(r)$ the number of sites inside the ball. In some sense these new couplings are dynamically generated. In the following we will discuss few interesting particular cases.

\section{TWO EXAMPLES}

Let us consider the standard nearest-neighbor Ising model with no external magnetic field, namely, we consider Eq. (4) with $J_{x, y}=J / 2$ for any $x, y \in \Lambda$ such that $|x-y|=1, J_{x, y}$ $=0$ otherwise, and $h_{x}=0$ for any $x \in \Lambda$. The Hamiltonian $H^{\prime}$ is the sum of averages performed over the four site crosses centered at each site of the lattice. We then expect all the possible interactions inside the cross.

As it as been seen in [13] it is possible to extract the potentials and rewrite the Hamiltonian $H^{\prime}$ in the following way:

$$
\begin{aligned}
H^{\prime}(\sigma)= & -J_{1} \sum_{\langle x y\rangle_{\sqrt{2}}} \sigma(x) \sigma(y)-J_{2} \sum_{\langle x y\rangle_{2}} \sigma(x) \sigma(y) \\
& -J_{3} \sum_{\diamond_{x y w z}} \sigma(x) \sigma(y) \sigma(w) \sigma(z),
\end{aligned}
$$

where the three sums [see Fig. 1(a)] are, respectively, performed over the pairs of next-to-nearest neighbors (sites at distance $\sqrt{2}$ ), the pairs of third neighbors (sites at distance 


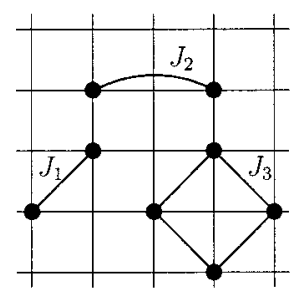

(a)

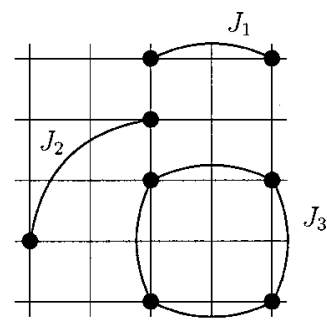

(b)
FIG. 1. Couplings $J_{1}, J_{2}$, and $J_{3}$ for the Hamiltonian (7) [Hamiltonian (9)] are shown in (a), (b).

2 ), and the four site diamond shaped clusters (plaquettes with side length equal to $\sqrt{2}$ ). The coupling constants are given by

$$
\begin{gathered}
J_{1}=\frac{1}{4} \log \cosh (2 \beta J) \sim \frac{1}{2} \beta J, \quad J_{2}=\frac{1}{2} J_{1}, \\
J_{3}=\frac{1}{16} \log \frac{\cosh ^{2}(2 \beta J)}{\cosh ^{8}(\beta J)} \sim-\frac{1}{4} \beta J,
\end{gathered}
$$

where " $\sim$ " means the limiting behavior for $\beta \rightarrow \infty$. There exist several possible ways to extract the potentials. A very natural one, in the case of spin variable, is to consider the function $\varphi_{x}(\sigma)=\log \cosh \left[(\beta J / 2) \Sigma_{y \in \Delta_{x}} \sigma(y)\right]$, where $\Delta_{x}$ $=\{y \in \Lambda:|y-x|=1\}$ is the set of nearest neighbors of site $x$, and its expansion $\varphi_{x}(\sigma)=\Sigma_{X \subset \Delta_{x}} c(X) \Pi_{y \in X} \sigma(y)$ with the coefficients $c(X)$ given by

$$
c(X)=\frac{1}{2^{\left|\Delta_{x}\right|}} \sum_{\sigma \in\{-1,+1\}^{\Delta_{x}}} \varphi_{x}(\sigma) \prod_{y \in X} \sigma(y)
$$

where, we recall, $\left|\Delta_{x}\right|=4$ is the cardinality of $\Delta_{x}$.

It is important to remark that the second nearest neighbor interaction, $J_{1}$, is positive and dominating; hence we expect a low temperature antiferromagnetic phase to exist. What appears interesting is that we have derived an antiferromagnetic behavior in a purely dynamical way as a result of the coupling proliferation. If a parallel heat bath Ising dynamics is implemented, the equilibrium Gibbs measure shows a low temperature antiferromagnetic phase despite the simple physical ferromagnetic coupling of the Ising model. This phenomenon is, obviously, absent if the Ising heat bath dynamics is implemented in a serial fashion.

The equilibrium properties of the model can be understood by remarking that two independent models are found if the lattice is partitioned into two square sublattices with step $\sqrt{2}$ (the even and the odd sublattice). Each model is, indeed, an eight vertex model with nearest neighbors coupling $J_{1}$, next-to-nearest neighbors coupling $J_{2}$, and plaquette interaction $J_{3}$. This model has been widely studied both in two $[14,15]$ and three $[16,17]$ dimensions. From Eq. (8) and the very well known properties of the two-dimensional eight vertex model we have that on each sublattice there are two coexisting low temperature phases, respectively with positive

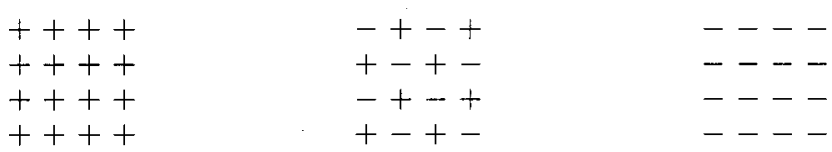

FIG. 2. The three ground states $\psi_{0}, \psi_{1}, \psi_{2}$ depicted from the left to the right.

and negative magnetization. Hence, by combining in all the possible ways the two phases we get, for the original model, the three different low temperature phases corresponding to the three ground states $\psi_{0}, \psi_{1}, \psi_{2}$ (see Fig. 2).

It is of some interest a direct study of the Hamiltonian (7): ground states can be defined as those configurations on which the Gibbs measure $\mu^{\prime}$, associated to the Hamiltonian $H^{\prime}$, is concentrated when the limit $\beta \rightarrow \infty$ is considered, namely as the minima of the energy $E(\sigma)$ $=\lim _{\beta \rightarrow \infty} H^{\prime}(\sigma) / \beta=-\Sigma_{x \in \Lambda}\left|S_{x}(\sigma)\right|$ uniformly in $\sigma \in \mathcal{S}$. It is rather clear that with periodic boundary conditions there exist three coexisting minima $\psi_{0}, \psi_{1}, \psi_{2} \in \mathcal{S}$ (see Fig. $2)$, with energy $-4|\Lambda|$, such that $\psi_{0}(x)=+1, \quad \psi_{1}(x)$ $=(-1)^{x_{1}+x_{2}}$, and $\psi_{2}(x)=-1$ for all $x=\left(x_{1}, x_{2}\right) \in \Lambda$. Notice that $\psi_{1}$ is the chessboard configuration.

The problem is, now, to understand if this coexistence of different states persists at a finite small temperature, namely, if the system undergoes a low temperature phase transition. We give an heuristic argument: at finite temperature ground states are perturbed because small droplets of different phases show up. The idea is to calculate the energetic cost of a perturbation of one of the four coexisting states via the formation of a square droplet of a different phase. A simple calculation, see [13], shows that the energy cost of a square droplet of side length $n$ of one of the two homogeneous ground states plunged in one of the two chessboards (or vice versa) is equal to $8 n$. On the other hand, if an homogeneous phase is perturbed as above by the other homogeneous phase, or one of the two chessboards is perturbed by the other one, then the energy cost is $16 n$. Hence, from the energetical point of view the most convenient excitations are those in which an homogeneous phase is perturbed by a chessboard or vice versa. Moreover, for each state $\psi_{0}, \psi_{1}, \psi_{2}$ there exist two possible energetically convenient excitations: there is no entropic reason to prefer one of the four ground states to the others when a finite low temperature is considered. This remark indicates that at small finite temperature the three ground states still coexist.

Let us consider, now, the Ising model with no external magnetic field and next-to-nearest neighbor interaction, namely, we consider Eq. (4) with $J_{x, y}=J / 2$ for any $x, y \in \Lambda$ such that $|x-y|=\sqrt{2}, J_{x, y}=0$ otherwise, and $h_{x}=0$ for any $x \in \Lambda$. It is possible to extract the potentials as seen above. The Hamiltonian $H^{\prime}$ can be written as

$$
\begin{aligned}
H^{\prime}(\sigma)= & -J_{1} \sum_{\langle x y\rangle_{2}} \sigma(x) \sigma(y)-J_{2} \sum_{\langle x y\rangle_{2} \sqrt{2}} \sigma(x) \sigma(y) \\
& -J_{3} \sum_{\square_{x y w z}} \sigma(x) \sigma(y) \sigma(w) \sigma(z),
\end{aligned}
$$




$$
\begin{array}{llll}
++++ & ++++ & +-+- & +-+- \\
-+-+ & ---- & +-+- & ---- \\
++++ & ++++ & +-+- & +-+- \\
-+-+ & ---- & +-+- & ----
\end{array}
$$

FIG. 3. The four ground states $\psi_{3}, \psi_{4}, \psi_{5}, \psi_{6}$ depicted from the left to the right.

where the three sums [see Fig. 1(b)] are, respectively, performed over the pairs of third nearest neighbors (sites at distance 2), the pairs of sites at distance $2 \sqrt{2}$, the plaquettes with side length equal to 2 . The coupling constants are still given by Eq. (8).

In order to study this model we remark that if the lattice is partitioned into four square sublattices with step 2, then we obtain four independent models one on each sublattice, each model being again an eight vertex model with nearest neighbors coupling $J_{1}$, next-to-nearest neighbors coupling $J_{2}$, and plaquette interaction $J_{3}$. Hence, on each sublattice we have the two degenerate ground states with all the spins, respectively, equal to one and minus one. By combining in all the possible ways these two states we get, for our model, $2^{4}$ $=16$ different ground states. On the torus, namely, when periodic boundary conditions are considered, some of the ground states are equivalent, so we get the seven states $\psi_{0}, \psi_{1}, \ldots, \psi_{6}$ in Figs. 2 and 3 . The fact that the phase transition persists at finite small temperature is, as seen above, a straightforward consequence of the known behavior of the eight vertex model.

In this paper we observed the relation between the two body ferromagnetic interaction of the Ising model and the low temperature antiferromagnetic behavior of the equilibrium Hamiltonian obtained evolving the initial system with a parallel heat bath dynamics. Any lattice system with two body interactions and having a self-organization resembling the parallel dynamics here described in the simple Ising model, could evolve towards different equilibrium states. The role of the parallel dynamics has been studied also in connection with the Ising-like transitions in coupled map lattices (CML's). CML's are lattices of interacting dynamical systems with continuous phase space (differently from PCA's that have discrete phase space) and discrete time. It has been shown that the nature of the update (synchronous or asynchronous) is a relevant parameter: continuous transitions of two-dimensional coupled chaotic [18] and stochastic [19] CML's with Ising-like discrete broken symmetry belong to a new universality class. A study on Toom cellular automata [20] seems to indicate that these particular examples of cellular automata belong to the same universality class of CML's with synchronized dynamics.

The evolution of the system we consider is governed by local energy (instead of local dynamical rules such as the Toom rule mentioned before) like in usual algorithms to simulate Ising interactions, but we consider synchronous updating. Also our results indicate that the influence of the updating nature on the physical properties of the equilibrium measure, is certainly an interesting feature that deserves further explorations.

\section{ACKNOWLEDGMENTS}

E.N.M.C. wishes to express his thanks to the Physics Department of the University of Helsinki for its warm hospitality. E.N.M.C. also thanks J. L. Lebowitz for having introduced him to the interesting topic of PCA's and P. Dai Pra for a useful discussion. A.D.P. acknowledges support from the EU-TMR program, Contract No. CT98-0169.
[1] O.N. Stavskaja, Math. USSR Sobrnik 21, 395 (1973).

[2] O. Kozlov and N. Vasilyev, Adv. Probab. Rel. Topics 6, 451 (1980).

[3] N. Vasiljev, Dev. Statistics 1, 99 (1978).

[4] M. Creutz, e-print hep-lat/9611017.

[5] J. Chalupa, P.L. Leath, and G.R. Reich, J. Phys. C 12, L31 (1979).

[6] P.M. Kogut and P.L. Leath, J. Phys. C 14, 3187 (1981).

[7] J. Adler and A. Aharony, J. Phys. A 21, 1387 (1998).

[8] M. Aizenman and J.L. Lebowitz, J. Phys. A 21, 3801 (1998); see also R. Cerf and E.N.M. Cirillo, Ann. Prob. 27, 1833 (1999) for an account on bootstrap percolation on a threedimensional lattice.

[9] G.Y. Vichniac, Phys. Rev. D 10, 96 (1984); S. Wolfram, Rev. Mod. Phys. 55, 601 (1983).

[10] P. Rujan, J. Stat. Phys. 49, 139 (1987); J.L. Lebowitz, C. Maes, and E. Speer, ibid. 59, 117 (1990).
[11] B. Derrida, in Fundamental Problem in Statistical Mechanics, edited by H. van Beijeren (Elsevier, New York, 1990), Vol. VII.

[12] S. Bigelis, E.N.M. Cirillo, J.L. Lebowitz, and E.R. Speer, Phys. Rev. E 59, 3935 (1999).

[13] E.N.M. Cirillo and F.R. Nardi (unpublished).

[14] R.J. Baxter, Exactly Solved Models in Statistical Mechanics (Academic Press, London, 1982).

[15] M. Nauenberg and B. Nienhuis, Phys. Rev. Lett. 33, 944 (1974).

[16] A. Cappi, P. Colangelo, G. Gonnella, and A. Maritan, Nucl. Phys. B 370, 659 (1992).

[17] E.N.M. Cirillo and G. Gonnella, J. Phys. A 28, 867 (1995).

[18] P. Marcq, H. Chaté, and P. Manneville, Phys. Rev. Lett. 77, 4003 (1996); Phys. Rev. E 55, 2606 (1997).

[19] F. Sastre and G. Pérez, Phys. Rev. E 64, 016207 (2001).

[20] D. Makowiec, Phys. Rev. E 60, 3787 (1999). 